ROME, ITALY

\title{
Design and Construction of the Mechanical Structure for Thin-Gap RPC Triplets for the Upgrade of the ATLAS Muon Spectrometer
}

\author{
Oliver Kortner ${ }^{a}$ on behalf of the ATLAS Muon Collaboration \\ ${ }^{a}$ Max-Planck-Institut für Physik, Föhringer Ring 6, 80805 München, Germany \\ E-mail: Oliver.Kortner@CERN.CH
}

\begin{abstract}
The advent of thin-gap RPCs with $1 \mathrm{~mm}$ gas gaps instead of $2 \mathrm{~mm}$ in the present RPCs opened the possibility to instrument the inner barrel layer of the ATLAS muon spectrometer where there is very limited amount of space in radial direction from the beam line. The environment is particularly dense in the barrel end-cap transition region. A compact mechanical structure coping with the expected thickness variations of the assembled RPCs is needed to fit into the limited available space. At the same time the mechanical structure must be sufficiently rigid to keep the deformations of the RPC packages within the allowed envelopes. The tight space constraints make it impossible to achieve the required rigidity with thick paper honeycomb plates as used in the present ATLAS RPCs. For the phase I upgrade of the barrel end-cap transition region of the ATLAS muon spectrometer three $1 \mathrm{~mm}$ gap RPCs are put into an aluminium frame. In this frame the RPC triplet is compressed with pre-bent $2 \mathrm{~mm}$ thick aluminium plates. The rigidity of the frame is achieved by stiffening rods connecting the lateral structure of the frame. The same concept can be used for the phase II upgrade of the inner layer of the muon spectrometer.
\end{abstract}

KeYwORDs: Gaseous detectors, resistive-plate chambers, trigger detectors.

${ }^{1}$ Corresponding author. 


\section{Contents}

1 Introduction 1

2 Challenges for the BI RPC upgrade 2

3 Structure of BI RPC singlets 3

4 Mechanical structure for BIS-78 RPC triplets 4

5 Summary 6

\section{Introduction}

Within less than a decade from now, CERN's Large Hadron Collider (LHC) will be upgraded to the so-called "High-Luminosity LHC" (HL-LHC) which will deliver an integrated luminosity of $3 \mathrm{ab}^{-1}$ to the ATLAS and CMS experiments during 10 years of operation. This will enable precision tests of the Standard Model of the electroweak and strong interactions, in particular, its Higgs sector and will maximize the potential to find physics processes beyond the Standard Model. The tenfold increase of the collider's integrated luminosity is achieved by an almost tenfold increase of the instantaneous luminosity which requires major upgrades of the LHC experiments.

The upgrade of the ATLAS detector's muon spectrometer is aimed at maintaining the efficiency and selectivity of its current muon trigger.

At the LHC the ATLAS muon spectrometer uses three layers of muon chambers operated in a magnetic field created by an air-core toroid system to trigger on muons with high transverse momenta $p_{\mathrm{T}}$ up to a pseudorapidity $|\eta|=2.4$ and to measure $p_{\mathrm{T}}$ with $4 \%$ resolution in a wide momentum range and $10 \%$ at $p_{\mathrm{T}}=1 \mathrm{TeV}$ up to $|\eta|=2.7$ [1]. Resistive Plate Chambers (RPC) in the barrel region and Thin Gap Chambers (TGC) in the end-cap regions with excellent time resolution of a few nanoseconds for $p p$ bunch crossing identification, but moderate spatial resolution, are used for the $1^{s t}$ level trigger. The high muon momentum resolution is achived by a precise measurement of muon trajectories with Cathode Strip Chambers (CSC) in the inner end-cap disk at large rapidities and Monitored Drift Tube (MDT) chambers in the rest of the spectrometer. These chambers have spatial resolutions better than $40 \mu \mathrm{m}$.

ATLAS uses a 2-level trigger system consisting of a hardware based $1^{\text {st }}$-level and a software based $2^{\text {nd }}$ level. A high- $p_{\mathrm{T}}$ muon trigger, built out of a conincidence of three RPCs in the barrel toroid magnet, and three TGCs behind the end-cap toroid magnet is part of the $1^{\text {st }}$ trigger level. The muon momentum is estimated from the sizes of the deviations of the trigger chamber hits from a straight line from the $p p$ interaction point.

Studies of the $1^{s t}$ level muon trigger in LHC Run 1 data showed that the trigger rate is dominated by triggers in the end-cap regions. A large fraction of these triggers are caused by charged particles, 
mainly protons, emerging from the radiation shielding and the materials of the end-cap toroid into the spectrometer. They leave traces in the TGCs behind the toroid magnet which look like high- $p_{\mathrm{T}}$ muons from the interaction point. These fake triggers can be rejected by taking trigger chambers in front of the end-cap toroid magnet into the $1^{s t}$ level concidence. After the removal of fake triggers the main source of muon triggers are muons with $p_{\mathrm{T}}<10 \mathrm{GeV}$ which are selected due to the poor momentum resolution at level 1 caused by the moderate spatial resolution of the trigger chambers. These will be rejected at the HL-LHC by the use of the precision muon chambers in the $1^{s t}$ level trigger.

In the phase-I upgrade of the ATLAS muon spectrometer during the long shutdown 2 of the LHC in 2019-2020 the inner end-cap wheels will be replaced by new wheels with improved trigger capabilities to minimize the fake muon trigger rate and increase the selectivity of the forward muon trigger. A part of the muons triggered in the end-cap system do not traverse these new wheels, but the end of the innermost barrel chamber layers. In order to reject fake muon triggers also in this region, stations of thin-gap RPCs and small-diameter muon drift-tube (sMDT) chambers will be installed during the long shutdown 2 .

This upgrade is a pilot to a larger upgrade in which new muon stations of thin-gap RPCs and small-diameter muon drift-tube chambers will be installed within the full pseudorapidity coverage of the barrel in order to close gaps in the acceptance of the current $1^{s t}$-level barrel muon trigger and to mitigate efficiency losses in the current RPC chambers at high background rates. This upgrade called "BI RPC upgrade" in this note is planned for the long shutdown between the end of the LHC and the start of the HL-LHC. [2] The pilot project will be referred to as BIS-78 project in the following.

\section{Challenges for the BI RPC upgrade}

In the current ATLAS detector the inner layer of the barrel muon spectrometer is only equipped with MDT precision muon chambers. In half of the barrel, in the so-called "small sectors", these leave no space for additional trigger chambers. They will therefore have to be replaced by sMDT precision muon chambers which occupy only half the space in radial direction than the MDT chambers. The liberated space will be equipped with a triplet of thin-gap RPCs.

Figure 1 shows a technical drawing of the end of the inner layer of the ATLAS muon spectrometer after the upgrade with a new station of an sMDT chamber and a thin-gap RPC. It illustrates the tight space constraints which require a very compact mechanical structure with high mechanical rigidity in order to avoid conflicts with the SMDT chamber.

The current ATLAS RPCs have a double-gap structure (see Figure 2) in which a $50 \mathrm{~mm}$ thick honeycomb structure provides the mechanical rigidity. The entire structure has a thickness of $92 \mathrm{~mm}$ exceeding the available space of $60 \mathrm{~mm}$ in the inner barrel layer.

In order to be able to install a triplet of RPC gas gaps within only $60 \mathrm{~mm}$ radial space three main development tasks had to be met:

- Use of thin-gap RPCs with $1 \mathrm{~mm}$ instead of $2 \mathrm{~mm}$ gaps;

- Each gap is operated by as autonomous RPC, a so-called "RPC singlet" which can be assembled without an external Faraday cage; 


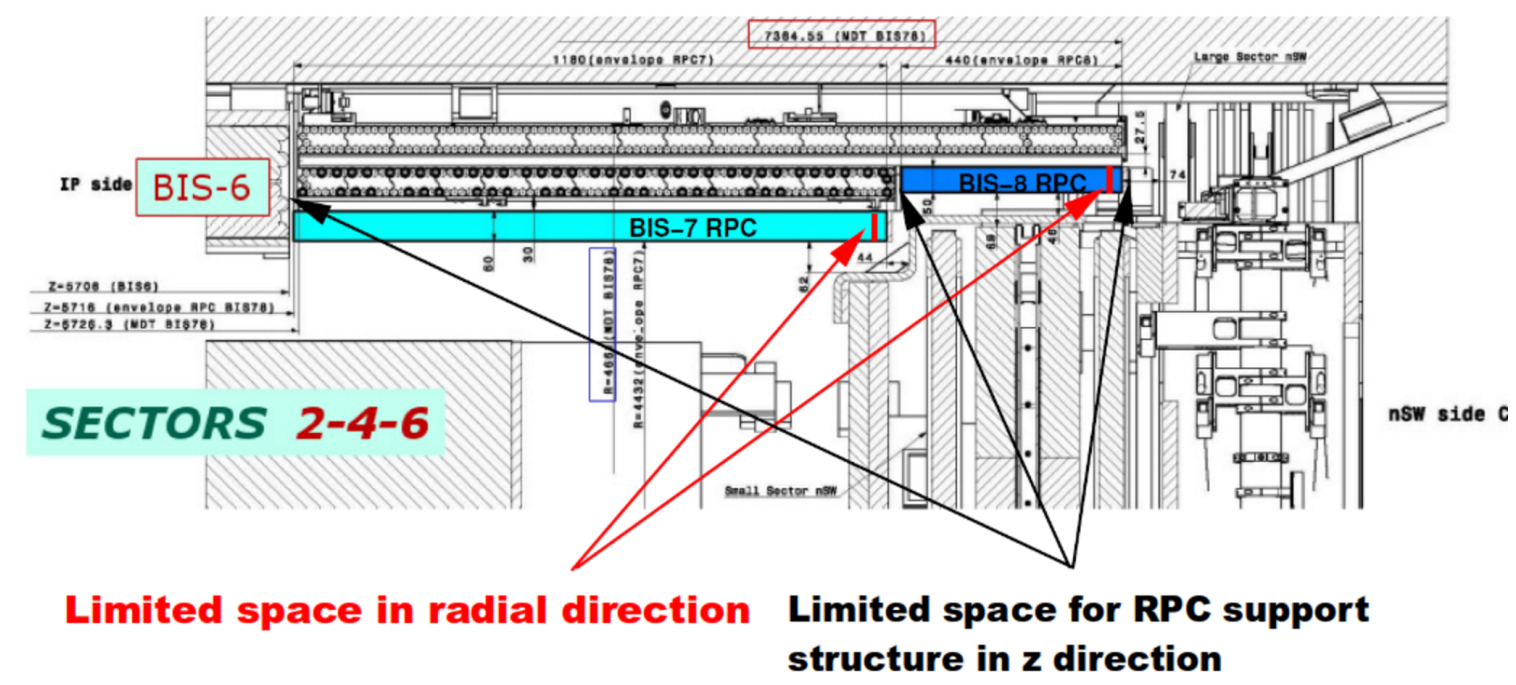

Figure 1. Technical drawing of the end of the inner layer of the ATLAS muon spectrometer with a new station of an sMDT chamber and thin-gap RPC. The new station has to fit into the very limited space which is currently occupied by MDT precision muon chambers, but no trigger chambers.

Copyright 2020 CERN for the benefit of the ATLAS Collaboration. CC-BY-4.0 license.

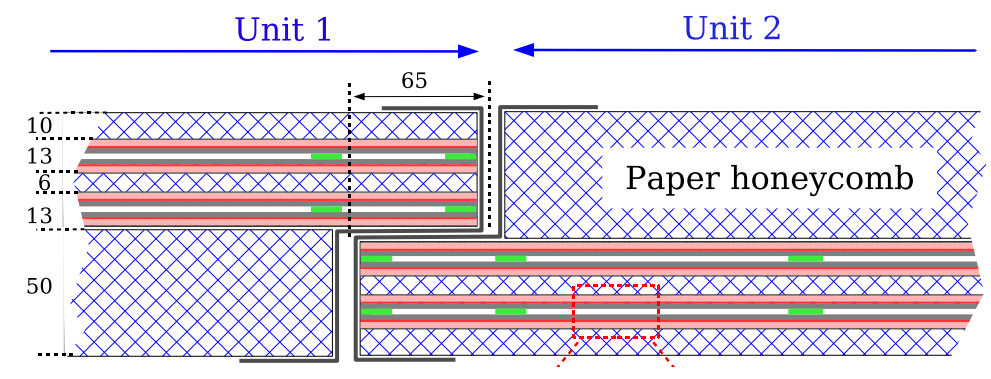

Figure 2. Structure of the current ATLAS RPCs. A doublet of $2 \mathrm{~mm}$ RPC gaps are used as active elements. The mechanical rigidity of the structure is provided by a $50 \mathrm{~mm}$ thick paper honeycomb. [1] Copyright 2020 CERN for the benefit of the ATLAS Collaboration. CC-BY-4.0 license.

- A rigid mechanical structure to support the RPC triplet independently of the accompanying sMDT chamber;

- A rigid mechanical frame for the three RPC singlets which stays within the tight $60 \mathrm{~mm}$ envelope including its deformations under gravity.

\section{Structure of BI RPC singlets}

A sketch of the cross section of a thin-gap RPC singlet is presented in Figure 3. The core of the singlet is the $1 \mathrm{~mm}$ gas gap between two high-pressure phenolic laminate plates. Measurements of protoype gas gaps had an average thickness of $4.2 \mathrm{~mm}$ with a variation of $\pm 0.2 \mathrm{~mm}$. In order to detect muon signals, so-called "read-out panels", with about $20 \mathrm{~mm}$ wide copper pick-up strips 
on the $0.3 \mathrm{~mm}$ printed circuit boards, are used. They are placed on the side attached to the gas gap structure, separated by a $3 \mathrm{~mm}$ thick dielectric made of FOREX ${ }^{1}$ from a copper ground plane on a $0.3 \mathrm{~mm}$ thick PCB. The RPC singlet is inclosed in its own Faraday cage consisting of the copper back plates of the read-out panels and an attached lateral copper foil. FOREX PVC foam was chosen as dielectric instead of polyester foam in the old RPCs to guarante minimal thickness variations and increased rigidity of the read-out panels. The RPC singlet has a nominal thickness of $11.8 \mathrm{~mm}$ with a tolerance of $0.7 \mathrm{~mm}$ driven by the thickness variations of the gas gap strucure and the FOREX plates.

This number was confirmed on prototype singlets by putting them onto a granite table and measuring the heights of many points on its surface with a scanarm.

\begin{tabular}{|c|c|c|}
\hline \multirow{2}{*}{$\begin{array}{l}\text { Dielectric of the read-out panel } \\
\text { FOREX plate }\end{array}$} & Component & Thickness [mm] \\
\hline & Electrode PCB & 0.3 \\
\hline \multirow{2}{*}{ Gas gap structure } & FOREX plate & $3.00 \pm 0.25$ \\
\hline & Gas gap & $4.2 \pm 0.2$ \\
\hline \multirow{2}{*}{$\begin{array}{l}\text { Dielectric of the read-out panel } \\
\text { FOREX plate }\end{array}$} & PET foil & 0.2 \\
\hline & $\mathrm{Cu}$ foil & 0.1 \\
\hline
\end{tabular}

Figure 3. Sketch of the layers of a thin-gap RPC singlet with the thicknesses of its components. The total thicknes of the singlet is $(11.8 \pm 0.7) \mathrm{mm}$.

Copyright 2020 CERN for the benefit of the ATLAS Collaboration. CC-BY-4.0 license.

\section{Mechanical structure for BIS-78 RPC triplets}

Figure 4 shows a photograph of a thin-gap RPC triplet in the mechanical structure. The sides of the structure are $3 \mathrm{~mm}$ thick 1-shaped aluminium profiles with holes for the connectors to the read-out electronics. Two $100 \mathrm{~mm}$ wide u-profiles on the bottom serve as interfaces to the rail support presented below and stiffen the bottom of the frame. The top cover also uses $3 \mathrm{~mm}$ thick 1-shaped aluminium profiles laterally and 3 rectangular profiles as stiffening rods. The compression of the RPCs which is provided by a prebent honeycomb structure in the current ATLAS RPCs is achieved by $2 \mathrm{~mm}$ thick prebent aluminium plates to save space.

The mechanical deformation of the entire structure under its gravitational load was measured with a scanarm by lifting the structure from a granite table used as reference plane. A maximum sag of $2 \mathrm{~mm}$ was found in agreement with a finite element analysis of the structure.

Production BIS-78 RPC triplets were integrated successfully with their partner sMDT chambers. A photograph of the integration of one BIS-78 station is shown in Figure 5. Like in the ATLAS experiment the triplet RPC and the accompanying sMDT chamber are mounted on rails with independent supports. The RPC support consists of two step like rectangular aluminium bars which are held on the rails by four igus ${ }^{\circledR}$ bearings. The frame of the RPC triplet is put onto the support structure via the u-shaped profiles on the bottom of the mechanical frame. No conflicts

${ }^{1}$ FOREX is a trademark of $3 \mathrm{~A}$ Composites for rigid foamboards made of PVC. 


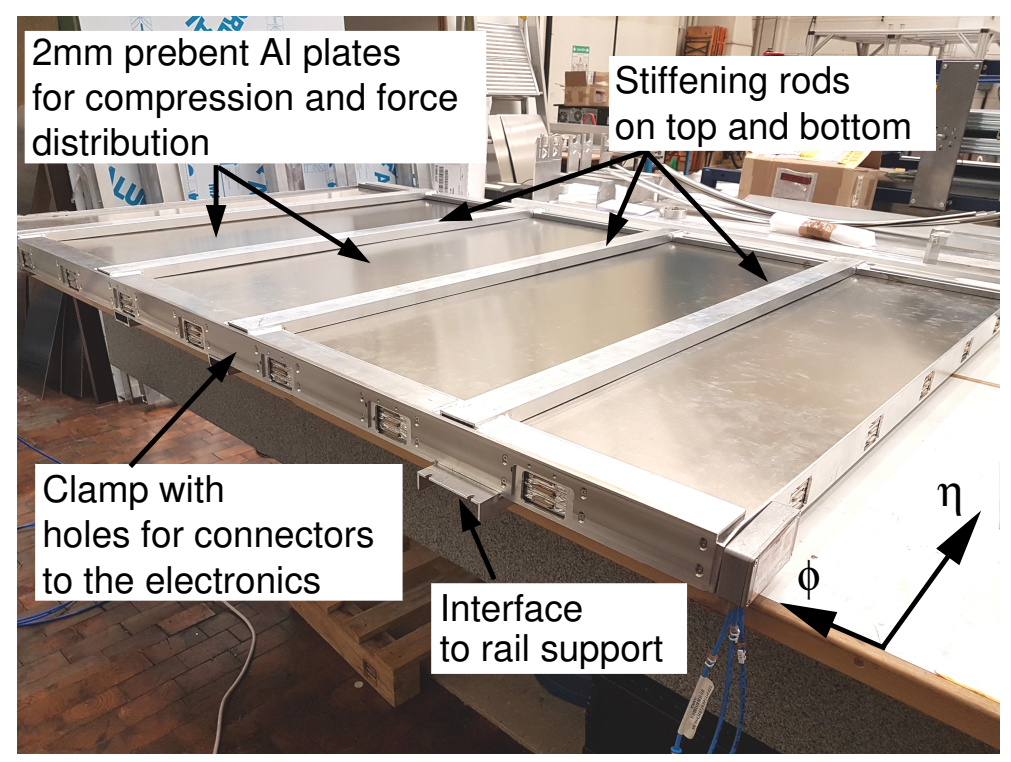

Figure 4. Photograph of the a thin-gap RPC triplet in its mechanical frame. Stiffening rods on the top and the $\mathrm{u}$-shaped interfaces to the rail support are responsible for the rigidity of the structure. The compression and force distribution across the surface of the RPCs is achieved with $2 \mathrm{~mm}$ thick prebent aluminium plates. Copyright 2020 CERN for the benefit of the ATLAS Collaboration. CC-BY-4.0 license.

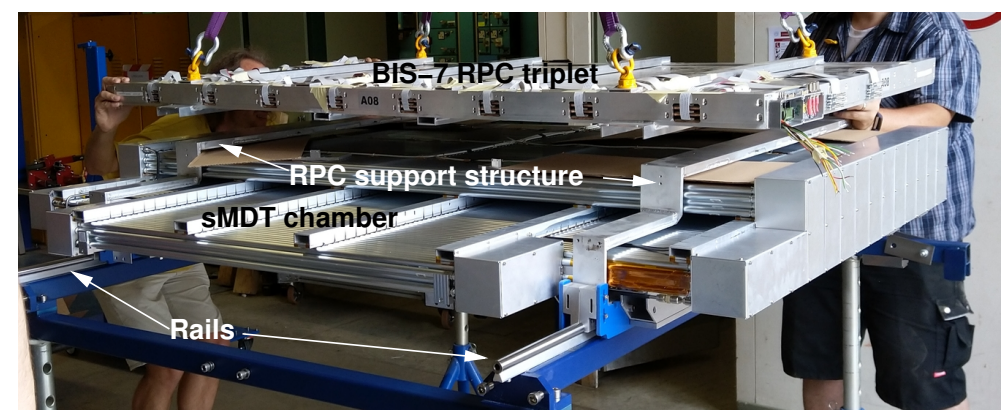

Figure 5. Photograph of the integration of a BIS-78 production RPC triplet with its accompanying sMDT chamber. The RPC triplet and the sMDT chamber sit on common rails, but on separate support structures. The RPC triplet is fixed to a step like support via the two u-shaped profiles on the bottom of the RPC frame. Copyright 2020 CERN for the benefit of the ATLAS Collaboration. CC-BY-4.0 license.

with the sMDT chamber were observed as expected from the finite element analysis predicting a deformation of RPC support bars by less than $2 \mathrm{~mm}$ under the full weight of the mechanical frame for the RPC triplet.

Figure 6 summarizes the structure of a BIS-78 RPC triplet in its mechanical frame in a crosssectional view. The entire structure has a nominal thickness of $55.4 \mathrm{~mm}$. Three RPC singlets have a thickness tolerance of $3 \cdot 0.7 \mathrm{~mm}=2.1 \mathrm{~mm}$. The plates of the frames have a thickness tolerance of $3.0 .3 \mathrm{~mm}=0.9 \mathrm{~mm}$. So the total thickness of the BI RPC triplet in its mechanical frame is less than $58.4 \mathrm{~mm}$ leaving $2 \mathrm{~mm}$ for the observed maximum gravitional sag. The design therefore complies 

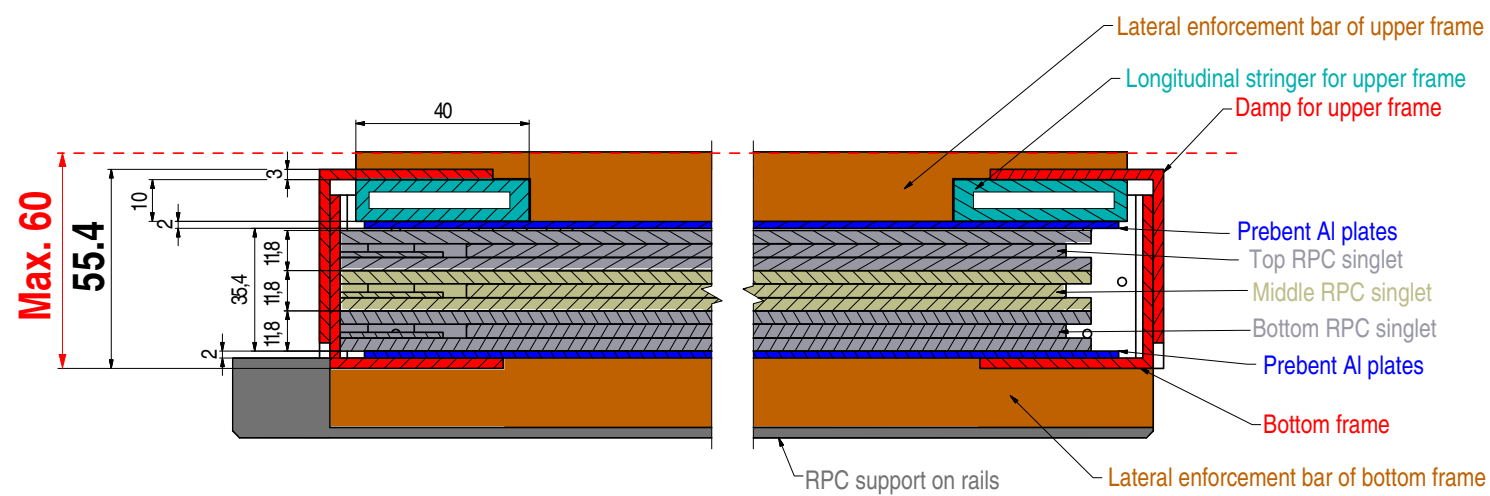

Figure 6. Cross section of a BIS-78 RPC triplet in its mechanical frame with the nominal thicknesses of the different components. The total nominal thickness of the entire structure is $55.4 \mathrm{~mm}$ fitting into the maximum available space of $60 \mathrm{~mm}$.

Copyright 2020 CERN for the benefit of the ATLAS Collaboration. CC-BY-4.0 license.

with the $60 \mathrm{~mm}$ envelope.

\section{Summary}

The inner layer of the barrel part of the ATLAS muon spectrometer will be instrumented with a triplet of thin-gap RPCs to close acceptance gaps and recuperate the expected efficiency losses of the present RPC system at the HL-LHC. In order to provide the required space for the RPCs the existing MDT chambers will be replaced by sMDT chambers. A mechanical structure for the thin-gap RPC triplets with a height of less than $60 \mathrm{~mm}$ and the required mechanical rigidity was successfully designed and produced for the upgrade of the ATLAS muon spectrometer.

\section{References}

[1] ATLAS Collaboration, The Atlas Experiment at the CERN Large Hadron Collider, JINST 3 (2008) S08003.

[2] ATLAS Collaboration, Technical Design Report for the Phase-II Upgrade of the ATLAS Muon Spectrometer, Tech. Rep. CERN-LHCC-2017-017. ATLAS-TDR-026, CERN, Geneva (Sep 2017). URL https : //cds . cern. ch/record/2285580 